\title{
MONOTONE DECOMPOSITIONS OF IUC CONTINUA
}

BY

\author{
W. DWAYNE COLLINS ${ }^{1}$
}

\begin{abstract}
For the class of hereditarily unicoherent metric continua a spectrum of monotone decompositions has been developed by several authors which "improves" the quotient spaces. This spectrum is developed for a broader class of continua, namely continua with property IUC. A metric continuum $M$ has property IUC provided each proper subcontinuum of $M$ with interior is unicoherent. One important result which develops is that semiaposyndetic IUC continua are hereditarily arcwise connected. Also the notion of smoothness is studied for IUC continua.
\end{abstract}

0. Introduction. In [5] FitzGerald and Swingle, by use of set functions, described a monotone upper semicontinuous decomposition $\mathscr{D}_{a}$ of a compact Hausdorff continuum $M$ such that $\mathscr{D}_{a}$ is the core decomposition of $M$ with respect to having an aposyndetic quotient space. A spectrum of decompositions which "fills in" between $M$ and $M / \mathscr{D}_{a}$ has been developed for hereditarily unicoherent metric continua by such authors as J. J. Charatonik [1], E. J. Vought [8, 12] and G. R. Gordh [6, 8].

The main purpose of this paper is to extend the spectrum of decompositions to the class of IUC continua. A compact metric continuum has property IUC provided each proper subcontinuum with interior is unicoherent [3]. If the continuum has property IUC hereditarily then the continuum has property HIUC. Note that the class of IUC continua includes all atriodic continua and hereditarily unicoherent continua.

Throughout this paper $M$ will denote a compact metric continuum. An upper semicontinuous (u.s.c.) decomposition $\mathscr{D}$ of $M$ is core with respect to some property $P$ provided $\mathscr{D}$ is the unique minimal decomposition with respect to which $\mathscr{D}$ has property $\mathrm{P}$. A set function $N$ is expansive provided that if $A$ and $B$ are subsets of $M$ then $A \subseteq N(A)$ and $N(A) \subseteq N(B)$ whenever $A \subseteq B$. The subset $A$ is $N$-closed provided $A=N(A)$. In [5, Theorem 2.5, p. 37] FitzGerald and Swingle prove that if $N$ is any expansive set function on $M$ then there exists a core decomposition $\mathcal{G}$ of $M$ with respect to the property: $\mathcal{G}$ is u.s.c with $N$-closed elements. They also note that if $N$ is monotone then $\mathcal{G}$ is monotone.

Let $\mathscr{D}$ be a u.s.c. decomposition of $M$ and $\mathscr{K} \subseteq \mathscr{D}$. We denote by $\mathscr{K} *$ the subset of $M$ consisting of the sum of the elements of $\mathcal{K}$. If $B$ is a subset of $M / \mathcal{D}$ then $B^{-1}$ will

Received by the editors April 10, 1983.

1980 Mathematics Subject Classification. Primary 54F20, 54B15.

Key words and phrases. Hereditarily decomposable, upper semicontinuous decompositions, property IUC, property HIUC, aposyndetic, semiaposyndetic, smooth.

'This paper comprises a portion of the author's dissertation in partial fulfillment of the doctoral degree at the University of Houston, Houston, Texas. 
denote $f^{-1}(B)$, where $f$ is the quotient map from $M$ onto $M / 01$. If $\mathscr{K} \subseteq$ 은 and $X \subseteq M$ then $\mathscr{K} \cap X$ will denote $\{k \cap X \mid k \in \mathscr{K}\}$, the restriction of $\mathcal{K}$ to $X$.

Finally, it should be noted that property IUC is preserved by monotone mappings, as this fact will be used throughout this paper.

I. Decompositions and hereditary decomposability. For $A \subseteq M$ let $V(A)$ denote the closure of the sum of $A$ and the indecomposable subcontinua of $M$ which intersect $A$. Now $V$ is expansive and montone. Let $\mathscr{D}_{V}$ denote the monotone u.s.c. core decomposition of $M$ into $V$-closed elements. Note that $M / D_{V}$ is hereditarily decomposable.

THEOREM 1.1. Suppose that $M$ has property HIUC and on is a monotone u.s.c decomposition of $M$. If $M / \mathcal{D}$ is hereditarily decomposable then each element of ol is $V$-closed.

Proof. Let $I$ be an indecomposable subcontinuum of $M$ and $\mathscr{T}=\{d \in(\mathbb{2}) \mid d$ intersects $I$ \}. If $\mathscr{K}$ is degenerate then $I$ is contained in the element of $\mathscr{K}$. So suppose that $\mathscr{K}$ is nondegenerate. Now $\mathcal{K} \cap I$ is a u.s.c. decomposition of $I$ and since $I /(\mathscr{K} \cap I)=\mathscr{K}^{*} / \mathscr{K}$ which is hereditarily decomposable, some element $d_{1}$ of $\mathscr{K}^{*}$ has a nonconnected intersection with $I$.

Let $d \in \mathscr{K}-\left\{d_{1}\right\}$ and suppose that $d^{-1} \not Z I$. Then $d_{1}^{-1} \cup I$ is a nonunicoherent proper subcontinuum of $d_{1}^{-1} \cup I \cup d^{-1}$ with interior respect to $d_{1}^{-1} \cup I \cup d^{-1}$, a contradiction. Hence $d^{-1} \subseteq I$ and each element of $\mathscr{K}-\left\{d_{1}\right\}$ must lie in a composant of $I$. Then by [4] either (1) $d_{1}^{-1}$ hits every composant of $I$ or (2) $d_{1}^{-1}$ misses uncountably many composants.

Suppose (1). By [4, Theorem 8, p. 40] some composant $C$ is hit by $d_{1}^{-1}$ in a nonconnected intersection. Let $A$ and $B$ be components of $C \cap d_{1}^{-1}$ and let $N$ be a subcontinuum of $C$ containing $A$ and $B$. Then $N \cup d_{1}^{-1}$ is a nonunicoherent proper subcontinuum of $I \cup d_{1}^{-1}$ with interior with respect to $I \cup d_{1}^{-1}$.

Suppose (2). Let $C$ be a composant of $I$ which misses $d_{1}^{-1}$. We show that $C /(\mathfrak{K} \cap C)$ is a composant of $I /(\mathscr{K} \cap I)$. Let $x$ and $z$ be points of $C /(\mathcal{K} \cap C)$ and $N$ be a subcontinuum of $C$ containing $x^{-1}$ and $z^{-1}$. Hence $f(N)$ is a subcontinuum of $C /(\mathcal{K} \cap C)$ containing $x$ and $z$, where $f$ is the induced quotient map from $M$ to $M / \mathscr{R}$. Let $y \in I /(\mathcal{K} \cap I)-C /(\mathfrak{K} \cap C)$ and $S$ be a subcontinuum of $I /(\mathscr{K} \cap I)$ from $x$ to $y$. Let $T$ be a component of $S-\left\{d_{1}\right\}$. Hence $\operatorname{cl}\left(T^{-1}\right)$, the closure of $T^{-1}$, is a subcontinuum of $I$ intersecting two composants and therefore $\operatorname{cl}\left(T^{-1}\right)=I$. Hence $S=I /(\mathfrak{K} \cap I)$ and $C /(\mathscr{K} \cap C)$ is a composant of $I /(\mathcal{K} \cap I)$.

Since there are uncountably many composants of $I$ missing $d_{1}^{-1}$ there are uncountably many composants of $I /(\mathcal{K} \cap I)$, a contradiction. Hence the theorem is established.

COROllaRy 1.2. If $M$ has property HIUC then $M$ admits a monotone core decomposition, $\mathcal{D}_{h d}$, with respect to the quotient space being hereditarily decomposable, and this decomposition is the same as $\mathscr{D}_{V}$.

One cannot weaken the hypothesis of Theorem 1.1 to IUC continua as will be seen in the following 
EXAMPLE 1.3. Let $K$ denote the sum of the Knaster indecomposable continuum and an $\operatorname{arc} A$ which intersects each composant. Let $M$ be the sum of $K$ and a half-ray which limits on $K$. Hence $M$ has property IUC but not HIUC. Let $\mathscr{D}$ be the monotone u.s.c. decomposition of $M$ induced by collapsing the $\operatorname{arc} A$ to a single point. Hence $M / \mathcal{O}$ is hereditarily decomposable, but $K$ fails to lie in a single element of Q 2 .

II. Decompositions and arcwise connectivity. If $I$ is an irreducible continuum there exists a monotone core decomposition $\mathcal{G}$ of $I$ such that $I / \mathcal{G}$ is an arc or degenerate. The elements of the decomposition $\mathcal{G}$ are called the layers of $I$.

Definition 2.1 (Charatonik). A monotone u.s.c. decomposition $\mathscr{D}$ of $M$ is admissible provided each layer of every irreducible subcontinuum of $M$ lies in an element of $\mathbb{Q}$.

If $A \subseteq M$ then $C(A)$ denotes the closure of the sum of all layers of irreducible subcontinua which intersect $A$. Clearly $C$ is expansive and monotone. Let $\mathscr{Q}_{C}$ denote the core decomposition of $M$ into $C$-closed elements. Hence $M / \mathscr{Q}_{C}$ is hereditarily arcwise connected since each layer of every irreducible subcontinuum lies in an element of $\mathscr{Q}_{C}$. Note that any decomposition of $M$ into $C$-closed elements is admissible.

THEOREM 2.2. If $M$ has property HIUC and 9 is a monotone u.s.c. decomposition of $M$ such that $M / \mathcal{D}$ is hereditarily arcwise connected, then each element of $\mathscr{D}$ is $C$-closed.

Proof. Let $I$ be an irreducible subcontinuum of $M$.

Case 1. I is its own layer. In [11] E. J. Vought described the structure of the layers of irreducible continua that have nonvoid interior. An outline of his technique follows.

Let $I$ be an irreducible continuum and $J$ be a layer of $I$ with interior. Let $I_{1}, I_{2}, \ldots$ denote the indecomposable subcontinua of $J$ with interior. Let $\mathrm{Ch}_{0}\left(I_{i}\right)=I_{i}$ for each positive integer $i$. For each nonlimit ordinal $\alpha$ define

$$
\mathrm{Ch}_{\alpha}\left(I_{i}\right)=\operatorname{cl}\left\{y \in J \mid y \text { can be } \mathrm{Ch}_{\alpha-1} \text {-chained to } I_{i}\right\}
$$

(if $F$ is a set function then $y$ can be $F$-chained to $R \subseteq J$ if there exists $I_{i_{1}}, I_{i_{2}}, \ldots, I_{i_{n}}$ such that $F\left(I_{i_{1}}\right), F\left(I_{i_{2}}\right), \ldots, F\left(I_{i_{n}}\right)$ is a simple chain where $y \in F\left(I_{i_{1}}\right)$ and $R$ intersects $\left.F\left(I_{i_{n}}\right)\right)$. If $\alpha$ is a limit ordinal, define $\mathrm{Ch}_{\alpha}\left(I_{i}\right)=\operatorname{cl}\left(\cup_{\beta<\alpha} \operatorname{Ch}_{\beta}\left(I_{i}\right)\right)$. Let $\gamma$ be a countable ordinal such that $\mathrm{Ch}_{\gamma}\left(I_{i}\right)=\mathrm{Ch}_{\gamma+1}\left(I_{i}\right)$ for all $i$. He then proves that $\operatorname{cl}\left(J^{0}\right)=\mathrm{Ch}_{\gamma}\left(I_{1}\right)$.

Hence we assume that $I=J$ and hence $I=\mathrm{Ch}_{\gamma}\left(I_{1}\right)$. Since $M / \mathcal{Q}$ is hereditarily arcwise connected it is hereditarily decomposable and, by Theorem 1.1, each indecomposable subcontinuum is contained in an element of $\mathscr{D}$ and for each ordinal $\beta \leqslant \gamma$ so is $\mathrm{Ch}_{\beta}\left(I_{1}\right)$.

Case 2. The collection of layers $\mathcal{L}$ of $I$ is nondegenerate. Let $\mathscr{K}=\{d \in \mathscr{Q} \mid d$ intersects $I\}$. Hence $\mathscr{K}^{*} / \mathcal{K}=I /(\mathscr{K} \cap I)$ is a hereditarily arcwise connected subcontinuum of $M / \mathscr{Q}$. If $\mathcal{K} \cap I$ is monotone, then $I /(\mathcal{K} \cap I)$ is irreducible and is hence either degenerate or an arc. But by [1, Corollary 8, p. 123] $\mathscr{K} \cap I$ is admissible, and hence 2 is also. 
So we suppose that $d_{1} \in \mathscr{K}$ such that $d_{1}^{-1} \cap I$ is not connected. As in the proof of Theorem 1.1 we assume that $d^{-1} \subseteq I$ for each $d \in \mathscr{K}-\left\{d_{1}\right\}$.

Let $\mathcal{L}=\left\{L_{x}\right\}$ where $L_{x}$ denotes the layer of $I$ coniaining the point $x$ of $I$. If $I$ is irreducible from the point $a$ to the point $b$ then $L^{*} / \mathcal{L}$ is an $\operatorname{arc}$ from $L_{a}$ to $L_{b}$. Denote by $\left[L_{a}, L_{b}\right]$ the $\operatorname{arc} L^{*} / \mathcal{L}$ in $M / \mathcal{Q} D$.

Note that (1) $d_{1}^{-1}$ intersects $L_{a}^{-1}$ and $L_{b}^{-1}$ and $d_{1}^{-1} \cap L_{x}^{-1}$ is a continuum for each $x$ in $I$. Since $I$ is irreducible and $d_{1}^{-1} \cap I$ is not connected, no component of $d_{1}^{-1} \cap I$ meets both $L_{a}^{-1}$ and $L_{b}^{-1}$.

Also $d_{1}^{-1} \cap I$ is the sum of two disjoint continua $A$ and $B$ where $A$ intersects $L_{a}^{-1}$ and $B$ intersects $L_{b}^{-1}$. For if $A$ and $B$ are components of $d_{1}^{-1} \cap I$ intersecting $L_{a}^{-1}$ and $L_{b}^{-1}$, respectively, then by (1) these are the only two such components. Suppose $C$ is a component of $d_{1}^{-1} \cap I$ different from $A$ and $B$. Then $C$ misses $L_{a}^{-1} \cup L_{b}^{-1}$. Let $L_{z}$ be in $\mathcal{L}$ such that $C$ intersects $\left[L_{a}, L_{z}\right]^{-1} \subseteq\left[L_{a}, L_{b}\right)^{-1}$. Hence $d_{1}^{-1} \cup\left[L_{a}, L_{z}\right]^{-1}$ is a nonunicoherent proper subcontinuum of $d_{1}^{-1} \cup I$ with interior with respect to $d_{1}^{-1} \cup I$. Likewise we may suppose that $A \subseteq L_{a}^{-1}$ and $B \subseteq L_{b}^{-1}$.

Let $K^{\prime}=\{C \mid C$ is a component of $\mathscr{K} \cap I\}$. We show that $I / K^{\prime}$ is arcwise connected. Let $p$ be a point of $\left(L_{a}, L_{b}\right)^{-1}$. Hence $\left[L_{a}, L_{p}\right]^{-1}$ and $\left[L_{p}, L_{b}\right]^{-1}$ are subcontinua of $I$ containing $p$ and, respectively, $A$ and $B$. Let $A_{p}$ and $B_{p}$ be arcs in $I /(\mathscr{K} \cap I)$ from the element containing $p$ to $d_{1} \cap I$ contained in $f\left(\left[L_{a}, L_{p}\right]^{-1}\right)$ and $f\left(\left[L_{p}, L_{b}\right]^{-1}\right)$, respectively, where $f$ is the induced quotient map for $\mathscr{K} \cap I$.

Now $A_{p}^{-1}$ cannot meet both $L_{a}^{-1}$ and $L_{b}^{-1}$ since $I$ is irreducible, and hence $A_{p}$ is an arc in $I / \mathscr{F}^{\prime}$ from the element containing $p$ to $A$. Likewise $B_{p}$ is an $\operatorname{arc}$ in $I / \mathcal{K}^{\prime}$ from $L_{p}$ to $B$. If $p$ is in $L_{a}^{-1}-A^{-1}$ then let $A_{p}$ be an arc in $I /(\mathcal{K} \cap I)$ from the element containing $p$ to $A$ contained in $f\left(L_{a}\right)$. Hence $A_{p}$ is in $I / \mathcal{K}^{\prime}$ and $I / \mathcal{K}^{\prime}$ is arcwise connected. But since $I / \mathscr{K}^{\prime}$ is irreducible, it is an arc. Therefore by [1], $\mathscr{K}^{\prime}$ is admissible and hence so is $\mathscr{K} \cap I$. Therefore $\mathscr{D}$ is admissible.

COROLlaRY 2.3. The HIUC continuum $M$ admits a core decomposition $\mathscr{D}_{\text {hac }}$ with respect to the quotient space being hereditarily arcwise connected, and this decomposition is exactly $\mathscr{O}_{C}$.

COROLlaRY 2.4. $\mathscr{D}_{V} \leqslant \mathscr{Q}_{C}$ and if $M$ has property HIUC then $\mathscr{Q}_{h d} \leqslant \mathscr{Q}_{\text {hac }}$, where " $\leqslant$ " means "refines".

Question 2.5. Is Theorem 2.2 true for continua with property IUC?

III. Decompositions and semiaposyndesis. The continuum $M$ is aposyndetic at the subset $A \subseteq M$ with respect to $B \subseteq M$ provided there exists a subcontinuum $H$ of $M$ containing $A$ in its interior that misses $B$. The continuum $M$ is aposyndetic provided $M$ is aposyndetic at each point with respect to every other point of $M$. Also, $M$ is semiaposyndetic provided for every two points of $M$, the continuum $M$ is aposyndetic at one of the points with respect to the other. Let $T(A)=A \cup\{p \in M \mid M$ is not aposyndetic at $p$ with respect to $A\}$ and $K(A)=A \cup\{p \in M \mid M$ is not aposyndetic at $A$ with respect to $p$. It is known that $T(A)$ is a continuum provided $A$ is connected, but this is not true for $K(A)$ in general.

LEMMA 3.1. If $X$ is an indecomposable subcontinuum of the IUC continuum $M$ then $X \subseteq K(x)$ for each $x$ in $X$. 
Proof. Let $x \in X$ and suppose there exists a point $w$ of $X$ and a subcontinuum $H$ of $M$ such that $x \in H^{0} \subseteq H \subseteq M-\{w\}$. Hence there exists a composant $C$ of $X$ and a component $K$ of $C-H$ such that $\operatorname{cl}(K) \cap H$ is not connected. But then $\operatorname{cl}(K) \cup H$ is a nonunicoherent proper subcontinuum of $M$ with interior, a contradiction. Hence $X \subseteq K(x)$.

THEOREM 3.2. If $M$ has property IUC and $x \in M$ then $K(x)$ is a subcontinuum of $M$.

Proof. Clearly $K(x)$ is closed. Suppose that $K(x)$ is the sum of two mutually exclusive point sets $A$ and $B$ with $x \in A$. Let $y \in B$ and $I$ be an irreducible subcontinuum of $M$ from $x$ to $y$. Hence $I$ contains a point $z$ not in $K(x)$. Let $H_{z}$ be a subcontinuum of $M$ such that $x \in H_{z}^{0} \subseteq H_{z} \subseteq M-\{z\}$. Hence $y \in H_{z}$ and $I \cap H_{z}$ is not connected. Since $M$ has property IUC we have that $M=I \cup H_{z}$.

Therefore by [3, Theorem 3.1] $M$ is nonunicoherent and by [3, Corollary 3.2] $M-I$ is connected. We show that $\operatorname{cl}(M-I)$ is indecomposable. Suppose rather that $\mathrm{cl}(M-I)$ is the sum of the proper subcontinua $C$ and $D$. Since $M$ has property IUC the set $C \cap D$ is a subcontinuum of $M$ which misses $I \cap H_{z}$. Let $\bigodot_{z}=\{k \mid k$ is a component of $H_{z}-\operatorname{cl}(M-I)$ whose closure intersects $\left.C\right\}$ and $\mathscr{D}_{z}=\{k \mid k$ is a component of $H_{z}-\operatorname{cl}(M-I)$ whose closure intersects $\left.D\right\}$. Since $M$ has property IUC the sets $\varrho_{z}^{*}$ and $\mathscr{Q}_{z}^{*}$ are mutually separated. Each of $C \cup \bigodot_{z}^{*}$ and $D \cup \mathscr{D}_{z}^{*}$ is a subcontinuum of $M$, and one must contain $x$ in its interior and miss $y$. Hence $\operatorname{cl}(M-I)$ is indecomposable, and by Lemma $3.1 \operatorname{cl}(M-I) \subseteq K(w)$ for each $w \in \operatorname{cl}(M-I)$.

If $x \in \operatorname{cl}(M-I)$ then $y \notin \operatorname{cl}(M-I)$ and each of $x$ and $y$ is in

$$
\operatorname{cl}(M-\operatorname{cl}(M-I)) \text {. }
$$

Since $I$ is irreducible, and by [3, Corollary 3.2] $M-\operatorname{cl}(M-I)$ is connected since $M$ is not unicoherent, we have that $I=\operatorname{cl}(M-\operatorname{cl}(M-I))$. Suppose that $I$ is the sum of the two proper subcontinua $E$ and $F$. Then $E \cap F$ misses $I \cap \operatorname{cl}(M-I)$ and we may assume that $x \in E-(E \cap F)$ and $y \in F-(E \cap F)$. Then $E \cup \operatorname{cl}(M-I)$ contains $x$ in its interior and misses $y$. Hence $I$ is indecomposable and $I \subseteq K(x)$, a contradiction.

If $x \notin \operatorname{cl}(M-I)$, by the above procedure we have that $\operatorname{cl}(M-\operatorname{cl}(M-I))$ is indecomposable and by Lemma 3.1 is in $K(x)$. But then $M=K(x)$, a contradiction. Hence $K(x)$ is a continuum.

COROllary 3.3. If $A$ is a subcontinuum of the IUC continuum $M$ then so is $K(A)$.

Definition 3.4. Let $Z(A)=T(A) \cap K(A)$. Hence $Z$ is an expansive set function.

LEMMA 3.5. Let $x$ be a point of the IUC continuum $M$. Then $Z(x)$ is a continuum.

Proof. Clearly $Z(x)$ is closed. Suppose that $Z(x)$ is the sum of two mutually exclusive closed sets $A$ and $B$. Since $M$ has property IUC the continuum $T(x) \cup K(x)$ is $M$ or has void interior.

Case 1. $M=T(x) \cup K(x)$. Hence $M$ is not unicoherent and by [3] $M-K(x)$ is connected. Let $y$ be a point of $M-K(x)$. Hence $y \in T(x)$ and $x \in \operatorname{cl}(M-K(x))$. 
As in the proof of Theorem $3.2 \mathrm{cl}(M-K(x))$ is indecomposable and hence $\operatorname{cl}(M-K(x)) \subseteq K(x)$, a contradiction.

Case 2. $T(x) \cup K(x)$ has void interior. We may suppose that $x \in A$ and let $y$ be a point of $B$. Let $I$ be a subcontinuum of $T(x)$ irreducible from $x$ to $y$, and $w$ be a point of $I-(I \cap K(x))$. Hence there exists a subcontinuum $H_{x}$ of $M$ such that $x \in H_{w}^{0} \subseteq H_{w} \subseteq M-\{w\}$. Now $I \cap H_{w}$ cannot be connected since $y \in H_{w}$ and $I$ is irreducible from $x$ to $y$. Hence $H_{w} \cup I$ is a nonunicoherent proper subcontinuum of $M$ with interior, a contradiction. Hence $Z(x)$ is a contiriuum.

COROllary 3.6. If $A$ is a subcontinuum of the IUC continuum $M$ then so is $Z(A)$.

Let $\mathscr{T}_{Z}$ denote the monotone core decomposition of $M$ with respect to having $Z$-closed elements.

THEOREM 3.7. If $M$ has property IUC then $M / \mathscr{Q}_{Z}$ is semiaposyndetic.

Proof. Let $d_{x}$ and $d_{y}$ be points of $M / \mathscr{D}_{Z}$. We will consider two cases.

Case 1. $T\left(d_{x}^{-1}\right)$ does not intersect $d_{y}^{-1}$. If $b \in d_{y}^{-1}$ there exists a continuum $H_{b}$ such that $b \in H_{b}^{0} \subseteq H_{b} \subseteq M-d_{x}^{-1}$. Since $d_{y}^{-1}$ is compact there exists a continuum $H$ such that $d_{y}^{-1} \subseteq H^{0} \subseteq H \subseteq M-d_{x}^{-1}$. Hence by the upper semicontinuity of $\mathscr{D}_{Z}$, $f(H)$ is a subcontinuum of $M / \mathscr{D}_{Z}$ containing $d_{y}$ in its interior and missing $d_{x}$ (where $f$ is the quotient map from $M$ to $M / \mathscr{Q}_{Z}$ ).

Case 2. $T\left(d_{x}^{-1}\right)$ intersects $d_{y}^{-1}$. We may suppose that $T\left(d_{y}^{-1}\right)$ intersects $d_{x}^{-1}$. Let $I$ be a subcontinuum of $T\left(d_{x}^{-1}\right)$ irreducible between $d_{x}^{-1}$ and $d_{y}^{-1}$, and let $b \in I \cap d_{y}^{-1}$. Hence $b \notin Z\left(d_{x}^{-1}\right)$ and $b \notin K\left(d_{x}^{-1}\right)$. There exists a subcontinuum $H_{b}$ of $M$ such that $d_{x}^{-1} \subseteq H_{b}^{0} \subseteq H_{b} \subseteq M-\{b\}$. Now $H_{b}$ intersects $d_{y}^{-1}$ but misses points of $I-$ $\left(I \cap d_{y}^{-1}\right)$ and hence $\left(H_{b} \cup d_{y}^{-1}\right) \cap I$ is not connected.

Since $M$ has property IUC we have $M=H_{b} \cup d_{y}^{-1} \cup I$ and by [3] $M$ is not unicoherent, and $Q=M-\left(d_{x}^{-1} \cup I \cup d_{y}^{-1}\right)$ is connected. Now bdy $(\operatorname{cl}(Q))$ is not connected and $\operatorname{cl}(Q)$ is irreducible about its boundary. If each of $I$ and $\operatorname{cl}(Q)$ is decomposable, then $I=I_{x} \cup I_{y}$ and $\operatorname{cl}(Q)=Q_{x} \cup Q_{y}$ where $I_{x} \cap I_{y}$ misses $d_{x}^{-1} \cup$ $d_{y}^{-1}$ and $Q_{x} \cap Q_{y}$ misses $\operatorname{bdy}(\operatorname{cl}(Q))$. We may assume that each of $I_{y}$ and $Q_{y}$ misses $d_{x}^{-1}$ since $M$ has property IUC. Therefore $d_{y}^{-1} \cup I_{y} \cup Q_{y}$ contains $b$ in its interior and misses $d_{x}^{-1}$, contradicting the fact that $b \in T\left(d_{x}^{-1}\right)$.

Suppose that $I$ is indecomposable. As in the proof of Theorem $3.2 b \in I \subseteq K\left(d_{x}^{-1}\right)$, a contradiction. Suppose $I$ is decomposable. Then $b \in Q$, for if not, $I_{y} \cup d_{y}^{-1}$ contradicts the fact that $b \in T\left(d_{x}^{-1}\right)$. Again $b \in Q \subseteq K\left(d_{x}^{-1}\right)$.

THEOREM 3.8. If $\mathscr{D}$ is a monotone u.s.c. decomposition of the IUC continuum M such that $M / \mathcal{Q}$ is semiaposyndetic then the elements of $\mathscr{Q}$ are Z-closed.

Proof. Let $d \in \mathscr{D}$ and since $Z$ is expansive $d^{-1} \subseteq Z\left(d^{-1}\right)$. Suppose there exists a point $w \in Z\left(d^{-1}\right)-d^{-1}$. Hence the element of $\mathscr{D}$ containing $w, d_{w}$, is different from $d$. Since $M / \mathcal{D}$ is semiaposyndetic there exists a subcontinuum of $M / \mathscr{D}$ containing one of $d$ and $d_{w}$ in its interior and missing the other point. Since $w \in K\left(d^{-1}\right)$ we may suppose that there is a subcontinuum $H$ of $M / \mathcal{D}$ such that $d_{w} \in H^{0} \subseteq H \subseteq$ $M / \mathscr{D}-\{d\}$. But then $H^{-1}$ is a subcontinuum of $M$ containing $w$ in its interior and 
missing $d^{-1}$, contradicting the fact that $w \in T\left(d^{-1}\right)$. Hence $Z\left(d^{-1}\right)=d^{-1}$ and the elements of $\mathscr{Q}_{2}$ are $Z$-closed.

Hence $M$ admits a monotone u.s.c. decomposition $\mathscr{O}_{s-a}$ which is core with respect to the quotient space being semiaposyndetic, and this decomposition is the core decomposition $\mathbb{O}_{Z}$. The next theorem enables one to extend the spectrum of decompositions.

THEOREM 3.9. If $A$ is a subset of the IUC continuum $M$ and $A=Z(A)$ then $A=C(A)$.

Proof. Since $C$ is expansive, $A \subseteq C(A)$. So suppose that $C(A) \nsubseteq A$. Let $L$ be a layer of the irreducible subcontinuum $I$ of $M$ such that $L^{-1}$ intersects $A$ but does not lie in $A$. We consider two cases:

(1) $L^{-1}$ has void interior with respect to $I$.

(2) $L^{-1}$ has nonvoid interior with respect to $I$.

Suppose (1). Denote by $[a, b]$ the quotient space of the decomposition of $I$ into layers. Let $w$ be a point of $L^{-1} \operatorname{such}$ that $w \in \operatorname{cl}\left([a, L)^{-1}\right) \cap \operatorname{cl}\left((L, b]^{-1}\right)$. Suppose that $w \in L^{-1}-A$. We show that $z \in Z(A)$.

Suppose there exists a subcontinuum $H$ of $M$ such that $w \in H^{0} \subseteq H \subseteq M-\{z\}$. Now $H \cap I$ is not connected, for if so, $H \cap I$ is a subcontinuum of $I$ containing $w$ in its interior with respect to $I$ which misses $z$. But in [10, Theorem 18, p. 26] Thomas showed that $I$ is not aposyndetic at $w$ with respect to $z$. Hence $H \cup I=M$ and $M$ is not unicoherent. But then $\operatorname{cl}(M-H)$ is connected and $z \in M-H \subseteq \operatorname{cl}(M-H)$ $\subseteq I-\{w\}$. But Thomas also showed that $I$ is not aposyndetic at $z$ with respect to $w$, and hence a contradiction. Therefore $z \in K(A)$. In like fashion it is shown that $z \in T(A)$ and hence $z \in Z(A)$, a contradiction.

If $w \in L^{-1}-A$ let $z \in A \cap L^{-1}$ and a contradiction is reached by similar arguments.

Suppose (2). Following Vought [11, pp. 75-77] there exists an indecomposable subcontinuum $N$ of $M$ which intersects $A, N \nsubseteq A$, and $N$ has interior with respect to $I$. Let $w$ be a point of $A \cap N$ and $z \in N-(A \cap N)$ where $z$ lies on a different composant of $N$. Suppose there exists a subcontinuum $H$ of $M$ such that $w \in H^{0} \subseteq$ $H \subseteq M-\{z\}$. Hence $H \cap N$ is not connected since $N$ is indecomposable, and therefore $M=H \cup N$ and $M$ is not unicoherent. But then $\operatorname{cl}(M-H)$ is a proper subcontinuum of $N$ with interior, a contradiction. Hence $z \in T(A)$. Likewise $z \in$ $K(A)$ and hence $z \in Z(A)=A$. Therefore $A=C(A)$.

COROLlary 3.10. $\mathscr{D}_{V} \leqslant \mathscr{D}_{C} \leqslant \mathscr{D}_{Z}$.

COROLLARY 3.11. If $M$ has property HIUC then $\mathscr{Q}_{h d} \leqslant \mathscr{D}_{h a c} \leqslant \mathscr{Q}_{s-a}$.

In what follows we generalize results in [6] known for hereditarily unicoherent continua.

COROLlARY 3.12. If the IUC continuum $M$ is semiaposyndetic then $M$ is hereditarily arcwise connected. 
Proof. Since $M$ is semiaposyndetic $M / \mathscr{Q}_{Z}=M$. But ${ }^{\mathscr{Q}} Q_{C} \leqslant \mathscr{O}_{Z}$ and hence $M / \mathscr{O}_{C}$ $=M / \mathcal{O}_{Z}=M$.

Therefore $M$ is hereditarily arcwise connected.

We now note several results concerning the types of quotient spaces of IUC continua.

THEOREM 3.13. If OD is a monotone u.s.c. decomposition of the IUC continuum $M$ such that $M / \mathcal{O})$ is semiaposyndetic then $M / \mathcal{O D}$ is a

(1) point,

(2) simple closed curve, or

(3) semiaposyndetic dendroid.

Proof. Suppose (1) and (2) are not true. Semiaposyndesis is preserved by monotone maps, and by Corollary $3.12 \mathrm{M} / \mathscr{Q}$ is hereditarily arcwise connected. Hence we need only show that $M / \mathscr{D}$ is hereditarily unicoherent.

Suppose not. Hence $M / \mathcal{Q}$ contains a simple closed curve $S$ and $S \neq M / \mathcal{Q}$. Let $x$ and $y$ be points of $S$. Since $M / \mathscr{D}$ is semiaposyndetic we assume there is a subcontinuum $H$ of $M / \mathscr{Q}$ such that $x \in H^{0} \subseteq H \subseteq M / \mathcal{Q}-\{y\}$. But $H \cup S$ is a nonunicoherent subcontinuum of $M / \mathscr{Q}$ with interior and hence $M / \mathscr{D}=H \cup S$. But then $S$ has interior, contradicting property IUC of $M / \mathcal{D}$.

COROLlaRY 3.14. If $\mathscr{D}$ is a monotone u.s.c. decomposition of the IUC continuum $M$ such that $M / \mathcal{O}$ is aposyndetic, then $M / \mathcal{O}$ is a

(1) point,

(2) simple closed curve, or

(3) dendrite.

IV. Decompositions and smoothness. Suppose that $M$ is a nonunicoherent IUC continuum. Then by the last paragraph of [3] $M$ is the sum of two irreducible subcontinua $A$ and $B$ such that $A=\operatorname{cl}(M-B)$ and $B=\operatorname{cl}(M-A)$. In [10] Thomas has shown that for an irreducible continuum $I$ the decomposition of $I$ into its layers is core with respect to the quotient space being aposyndetic. Hence $\mathscr{D}_{h a c}=\mathscr{Q}_{s-a}=\mathscr{D}_{a}$ and hence no further refining of the quotient space is possible. Hence likewise for $M$, since core decompositions of $M$ are unions of the respective core decompositions of $A$ and $B$. Therefore we need only consider the unicoherent IUC continua to complete the spectrum of decompositions.

The notion of smoothness was first studied by Charatonik and Eberhart [2] for dendroids and was later generalized in [7] for continua hereditarily unicoherent at a point. In [9] Maćkowiak generalized smoothness to metric continua. His definition follows.

DEFINITION 4.1 (MACOWIAK). A continuum $N$ is smooth at a point $p$ provided that if $x_{1}, x_{2}, x_{3}, \ldots$ is a sequence of points converging to the point $x$ of $N$ and $p x$ is an irreducible subcontinuum of $N$ from $p$ to $x$, then there exists a sequence $p x_{1}, p x_{2}, \ldots$ of continua, where $p x_{i}$ is irreducible from $p$ to $x_{i}$, such that the limiting set of $p x_{1}, p x_{2}, \ldots$ is $p x$. 
THEOREM 4.2 (MACKOWIAK). If $N$ is a continuum and $p$ is a point of $N$ then the following are equivalent:

(1) $N$ is smooth at $p$.

(2) For each subcontinuum $H$ of $N$ containing $p$ and for each open set $V$ containing $H$ there exists a continuum $K$ such that $H \subseteq K^{0} \subseteq K \subseteq V$.

He then notes that if $N$ is a continuum and $p$ is a point of $N$ then the statement that $N$ is smooth at $p$ implies the following property $(*)$ : if $y$ does not cut $x$ from $p$ then $N$ is aposyndetic at $x$ with respect to $y$. The point $y$ cuts $x$ from provided each subcontinuum of $N$ containing $x$ and $p$ must also contain $y$.

THEOREM 4.3. If $p$ is a point of the unicoherent IUC continuum $M$ then property (*) implies that $M$ is smooth at $p$.

Proof. Let $K$ be a subcontinuum of $M$ containing $p$ and let $V$ be an open set containing $K$. Let $z$ be a point on the boundary of $V$. For each point $w$ in $K$, the point $z$ does not cut $w$ from $p$, and hence there exists a continuum containing $w$ in its interior which misses $z$. By the compactness of $K$ we have a continuum $H_{z}$ such that $K \subseteq H_{z}^{0} \subseteq H_{z} \subseteq M-\{z\}$.

Hence $\left\{M-H_{z} \mid z \in\right.$ bdy $\left.V\right\}$ is an open cover of bdy $V$ and hence some finite subcollection $M-H_{z_{1}}, \ldots, M-H_{z_{n}}$ covers bdy $V$. But since $M$ is unicoherent and has property IUC the set $H_{z_{1}} \cap \cdots \cap H_{z_{n}}=H$ is a subcontinuum of $M$ and $K \subseteq H^{0} \subseteq H \subseteq V$.

Definition 4.4. Let $p$ be a point of $M$ and $A \subseteq M$. Then $p A$ denotes the intersection of all subcontinua of $M$ with interior which contain $p$ and $A$.

LEMMA 4.5. If $M$ is a unicoherent IUC continuum then $p A$ is a subcontinuum of $M$.

Proof. Now $p A$ is the intersection of a countable collection of continua $H_{1}, H_{2}, \ldots$, each having interior and containing $p$ and $A$. Let $K_{1}=H_{1}$ and if $i$ is a positive integer greater than 1 let $K_{i}=H_{i} \cap K_{i-1}$. Hence each $K_{i}$ is a subcontinuum of $M$ and $K_{1} \supseteq K_{2} \supseteq K_{3} \supseteq \cdots$. Hence $K=\bigcap_{i=1}^{\infty} K_{i}$ is a subcontinuum of $M$ containing $p$ and $A$ and therefore $K=p A$.

LEMma 4.6. If $p$ is a point of the continuum $M$ then the set function $p A$ is expansive.

Proof. Clearly $A \subseteq p A$ and if $A \subseteq B$ each subcontinuum containing $p$ and $B$ contains $A$.

The following definition is found in [8] for hereditarily unicoherent continua. We generalize the definition to unicoherent IUC continua. Note that the definition of $p A$ is different from the usual one, even for hereditarily unicoherent continua, and hence the following definition will not agree with that in [8].

Definition 4.7. Let $p$ be a point of the unicoherent IUC continuum $M$ and $A \subseteq M$. Define $T_{p}(A)=p A \cap T(A)$. Now $T_{p}$ is an expansive set function.

LEMMA 4.8. If $A$ is a subcontinuum of the unicoherent IUC continuum $M$ then so is $T_{p}(A)$. 
Proof. Suppose that $p A \cap T(A)$ is not connected. Let $x$ and $y$ be points of $p A \cap T(A)$ which lie on different components of $p A \cap T(A)$ and $I$ be a subcontinuum of $T(A)$ irreducible from $x$ to $y$. Let $z \in I-p A$ and $H$ be a subcontinuum of $M$ with interior such that $z \notin H$ and $p A \subseteq H$. But $H \cap I$ is connected, contradicting the irreducibility of $I$.

Let ${ }^{{ }^{1}} T_{p}$ denote the monotone core decomposition of $M$ into $T_{p}$-elements. Again note that if $\mathscr{Q} \Omega$ is a monotone u.s.c. decomposition of $M$ and $x \in M$ then $d_{x}$ denotes the element of $\mathscr{Q}$ which contains $x$.

THEOREM 4.9. If $M$ is a unicoherent IUC continuum then $M /{ }^{\text {OD }} T_{p}$ is smooth at $d_{p}$.

Proof. Suppose $x$ and $y$ are points of $M$ and $d_{y}$ does not cut $d_{x}$ from $d_{p}$ in $M /{ }^{\mathcal{O}}{ }_{T}$. We need only show that $T\left(d_{y}^{-1}\right)$ misses $d_{x}^{-1}$, for if it does, then there exists a subcontinuum $H$ of $M$ such that $d_{x}^{-1} \subseteq H^{0} \subseteq H \subseteq M-d_{y}^{-1}$. Then since ${ }^{\Omega_{2}} T_{p}$ is upper semicontinuous, $f(H)$ contains $d_{x}$ in its interior and misses $d_{v}$, where $f$ is the induced quotient map. But then $M / \mathcal{O}^{-} T_{p}$ is aposyndetic at $d_{x}$ with respect to $d_{y}$.

Hence suppose that $T\left(d_{y}^{-1}\right)$ intersects $d_{x}^{-1}$ and let $I$ be a subcontinuum of $M$ irreducible from $d_{p}^{-1}$ to $d_{x}^{-1}$ which misses $d_{y}^{-1}$. Now $d_{p}^{-1} \cup I \cup d_{x}^{-1} \cup T\left(d_{y}^{-1}\right)$ contains a subcontinuum $J$ irreducible from $p$ to $d_{y}^{-1}$. But $J$ contains a point $z$ of $T\left(d_{y}^{-1}\right)$ not in $d_{y}^{-1}$, and since $z \notin T_{p}\left(d_{y}^{-1}\right)$ we have $z \notin p d_{y}^{-1}$. There exists a subcontinuum $H$ of $M$ such that $H$ has interior, $z \notin H$, and $p d_{y}^{-1} \subseteq H$. By the irreducibility of $J$, the set $H \cap J$ is not connected, contradicting the fact that $M$ is a unicoherent IUC continuum.

THEOREM 4.10. If $M$ is a unicoherent IUC continuum, $M /{ }^{\mathscr{Q}} T_{p}$ is semiaposyndetic.

Proof. Let $d_{x}$ and $d_{y}$ be elements of $\mathscr{Q}_{T}$. If $T\left(d_{y}^{-1}\right)$ misses $d_{x}^{-1}$ then $M / \mathscr{Q} T_{p}$ is aposyndetic at $d_{x}$ with respect to $d_{y}$. Since by Theorem $4.9 M /{ }^{\circ} T_{p}$ is smooth at $d_{p}$ we may assume that $d_{x} \neq d_{p} \neq d_{y}$.

Suppose that $T\left(d_{y}^{-1}\right)$ intersects $d_{x}^{-1}$. Let $I$ be an irreducible subcontinuum of $T\left(d_{y}^{-1}\right)$ from $d_{y}^{-1}$ to $\dot{d}_{x}^{-1}$. Let $z$ be a point of $I \cap d_{x}^{-1}$. Since $z \notin d_{y}^{-1}$ and $z \in T\left(d_{y}^{-1}\right)$ then $z \notin p d_{y}^{-1}$. Hence there is a subcontinuum $H$ of $M$ with interior such that $p d_{y}^{-1} \subseteq H$ and $z \notin H$. Hence $H$ misses some point $w$ of $I-\left[\left(d_{x}^{-1} \cup d_{y}^{-1}\right) \cap I\right]$.

We may suppose further that $d_{x}$ cuts $d_{y}$ from $d_{p}$, for if not, since $M / \mathscr{Q}_{T_{p}}$ is smooth at $d_{p}$ the proof is complete. Hence $H$ intersects $d_{x}^{-1}$ and $\left(H \cup d_{x}^{-1}\right) \cup I$ is a subcontinuum of $M$ with interior and $\left(H \cup d_{x}^{-1}\right) \cap I$ is not connected since $w \notin H$ $\cup d_{x}^{-1}$. Therefore $T\left(d_{y}^{-1}\right)$ misses $d_{x}^{-1}$.

THEOREM 4.11. Suppose OD is a monotone u.s.c. decomposition of the unicoherent IUC continuum $M$ such that $M / \mathcal{D}$ is semiaposyndetic and smooth at $d_{p}$. Then each element of $\mathcal{D}$ is $T_{p}$-closed.

Proof. Let $x \in M$ and, since $T_{p}$ is expansive, $d_{x}^{-1} \subseteq T_{p}\left(d_{x}^{-1}\right)$. Suppose there exists a point $y$ in $T_{p}\left(d_{x}^{-1}\right)$ not in $d_{x}^{-1}$. Hence $d_{x} \neq d_{y}$ but $y \in p d_{x}^{-1} \cap T\left(d_{x}^{-1}\right)$. Since $y \in T\left(d_{x}^{-1}\right)$ we may suppose that $d_{x} \neq d_{p}$, for if $\dot{d}_{x}=d_{p}$, since $M / \mathscr{Q}$ is smooth at $d_{p}$, there exists a subcontinuum $H$ of $M$ such that $d_{x}^{-1} \subseteq H^{0} \subseteq H \subseteq M-d_{y}^{-1}$. Also $y$ can be chosen such that $y \notin d_{p}^{-1}$. 
Since $y \in T\left(d_{x}^{-1}\right)$ we have that $d_{x}$ cuts $d_{p}$ from $d_{y}$. Since $M / \mathcal{O D}$ is semiaposyndetic there exists a subcontinuum $K$ of $M$ such that $d_{x}^{-1} \subseteq K^{0} \subseteq K \subseteq M-d_{y}^{-1}$.

Consider $M-K$. Let $C$ be the component of $M-K$ containing $p$. Hence $\operatorname{cl}(C)$ misses $d_{y}^{-1}$ since $d_{x}$ cuts $d_{p}$ from $d_{y}$. But $\operatorname{cl}(C) \cup K$ is a subcontinuum with interior which contains $p$ and $d_{x}^{-1}$ missing $y$, a contradiction. Hence $d_{x}^{-1}=T_{p}\left(d_{x}^{-1}\right)$.

Hence there is a monotone core decomposition of the unicoherent IUC continuum $M, \mathscr{L}_{s}$, with respect to the quotient space being semiaposyndetic and smooth at the element containing $p$, and $\mathscr{D}_{s}=\mathscr{D}_{T_{p}}$.

COROLlaRY 4.12. $\mathscr{D}_{s-a} \leqslant \mathscr{Q}_{s} \leqslant \mathscr{D}_{a}$.

Proof. Since $M / \mathscr{Q}_{s}$ is semiaposyndetic, $\mathscr{Q}_{s-a} \leqslant \mathscr{Q}_{s}$. If $M / \mathscr{Q}_{a}$ is aposyndetic then clearly it is semiaposyndetic and property $(*)$ holds immediately.

Therefore we have the spectrum $\mathscr{Q}_{h d} \leqslant \mathscr{Q}_{h a c} \leqslant \mathscr{Q}_{s-a} \leqslant \mathscr{Q}_{s} \leqslant \mathscr{Q}_{a}$ for unicoherent HIUC continua and the spectrum $\mathscr{D}_{V} \leqslant \mathscr{D}_{C} \leqslant \mathscr{D}_{Z} \leqslant \mathscr{D}_{T_{P}} \leqslant \mathscr{Q}_{T}$ for unicoherent IUC continua, where $\mathscr{D}_{T}$ denotes FitzGerald and Swingle's core decomposition into $T$-closed elements.

\section{REFERENCES}

1. J. J. Charatonik, On decompositions of continua, Fund. Math. 79 (1973), 113-130.

2. J. J. Charatonik and C. Eberhart, On smooth dendroids, Fund. Math. 67 (1970), 297-322.

3. W. D. Collins, A property of atriodic continua, Illinois J. Math. (to appear).

4. H. Cook, On subsets of indecomposable continua, Colloq. Math. 13 (1964), 37-43.

5. R. W. FitzGerald and P. M. Swingle, Core decompositions of continua, Fund. Math. 61 (1967), 33-50.

6. C. R. Gordh, Jr., Concerning closed quasi-orders on hereditarily unicoherent continua, Fund. Math. 78 (1973), 61-73.

7. On decompositions of smooth continua, Fund. Math. 75 (1972), 51-60.

8. G. R. Gordh, Jr. and E. J. Vought, Monotone decompositions of hereditarily unicoherent continua via set functions and quasi-orders, Fund. Math. (to appear).

9. T. Maćkowiak, On smooth continua, Fund. Math. 85 (1974), 79-95.

10. E. S. Thomas, Jr., Monotone decompositions of irreducible continua, Dissertationes Math. (Rozprawy Mat.) 50 (1966), 1-74.

11. E. J. Vought, On decompositions of hereditarily unicoherent continua, Fund. Math. 102 (1979), 73-79. 12. $371-376$. , Monotone decompositions of Hausdorff continua, Proc. Amer. Math. Soc. 56 (1976),

Department of Mathematics, California State University, Chico, California 95929 